\title{
Disappearance of hepatic parenchymal nerves in human liver cirrhosis
}

\author{
J A Lee, Q Ahmed, J E Hines, A D Burt
}

\begin{abstract}
The normal human liver receives a substantial autonomic innervation that is thought to subserve motor, metabolic, and sensory functions. In this study an antibody to a neural axoplasmic protein (PGP 9.5) was used to visualise autonomic nerves in tissue from normal, precirrhotic, and cirrhotic livers. Nerve fibres were readily identified in the parenchyma and portal tracts of normal livers, and in those where the histological diagnosis was non-specific reactive hepatitis or acute liver injury. In precirrhotic and cirrhotic livers nerves in portal tracts and fibrous septae remained prominent, but the parenchymal innervation was reduced in precirrhotic livers and was absent from regenerating nodules in established cirrhosis. The causes and functional consequences of this parenchymal denervation in cirrhosis remain to be established.
\end{abstract}

The liver is innervated by both branches of the autonomic nervous system, receiving sympathetic input via the coeliac plexus and parasympathetic input mainly from the vagus. Most of these nerves enter the liver in association with blood vessels and bile ducts, and for many years it was assumed that motor functions related to these structures were the sole function of the hepatic nerves. It is now known, however, that in addition to blood vessels and bile ducts, the liver parenchyma also receives a substantial innervation in many species. Furthermore, there is now strong evidence that these hepatic parenchymal nerves have important metabolic and sensory, as well as motor functions. ${ }^{1-4}$ Metabolic effects include direct actions on carbohydrate and lipid metabolism, while sensory roles include osmoreception, baroreception, and possibly also responses to the concentrations of various metabolites present in portal blood. Although some species - for example the $\mathrm{rat}^{5}$ have only a scanty parenchymal innervation, the liver of primates,${ }^{6}$ including humans,${ }^{4}$ has a high density of parenchymal nerves, which may thus have important modulatory roles in normal liver function.

In developing and established cirrhosis, there are gross structural changes in liver tissue, as well as functional abnormalities. Since alterations in the hepatic innervation may have a role to play in these problems, we have investigated
Histological diagnoses

\begin{tabular}{lc}
\hline Diagnosis & No of cases \\
\hline Normal/non-specific reactive changes & 8 \\
Acute liver injury: & 6 \\
Extrahepatic biliary obstruction & 2 \\
Acute hepatitis & 5 \\
Intrahepatic cholestasis & 6 \\
Precirrhotic chronic liver disease: & 5 \\
Alcoholic hepatitis with perivenular fibrosis & \\
Primary biliary cirrhosis stage I or II & 6 \\
Established cirrhosis: & 7 \\
Alcoholic cirrhosis & 3 \\
Primary biliary cirrhosis & 2 \\
Haemochromatosis & 4 \\
Autoimmune liver disease & \\
Cryptogenic cirrhosis & 54 \\
Total & \\
\hline
\end{tabular}

the distribution of hepatic nerves in samples obtained from normal and diseased human livers. The results show that hepatic parenchymal nerves are reduced in precirrhotic liver disease and are absent from regenerating hepatic nodules in established cirrhosis.

\section{Methods}

SPECIMENS

Wedge biopsy specimens were obtained at laparotomy from patients with suspected liver disease. Tissue was fixed in $10 \%$ buffered formalin and processed for paraffin wax embedding. In some cases $(n=6)$ normal liver was obtained from cadaveric renal transplant donors. This tissue was perfusion fixed in $2 \%$ paraformaldehyde in $0.1 \mathrm{mmol} / 1$ phosphate buffer through vessel openings at the cut surface and was then processed for paraffin wax embedding. The histological diagnoses of the samples (obtained from sections stained with routine haematoxylin and eosin) are shown in the Table.

\section{IMMUNOHISTOCHEMISTRY}

A peroxidase-antiperoxidase technique was used with the anti-PGP 9.5 antibody. Deparaffinised sections $(3 \mu \mathrm{m})$ were treated in the following way: (a) incubated in $0.5 \%$ hydrogen peroxide in methanol; (b) washed in tap water, then incubated with $0 \cdot 1 \%$ porcine trypsin.(Sigma, $\mathrm{UK}$; T8128) in $0.1 \% \mathrm{CaCl}_{2}, \mathrm{pH} 7 \cdot 8,37^{\circ} \mathrm{C}$ for 10 minutes; (c) washed in tap water, then Tris buffered saline (TBS); (d) incubated with normal swine saline (NSS)/TBS for five minutes, 
Figure 1: Normal liver. (A) $P G P 9.5$ immunoreactive fibres and bundles are seen with this large portal tract (arrows) (magnification $\times 25$ ). (B) Abundant intrasinusoidal PGP 9.5 immunoreactive fibres are seen (magnification $\times 150$ ).

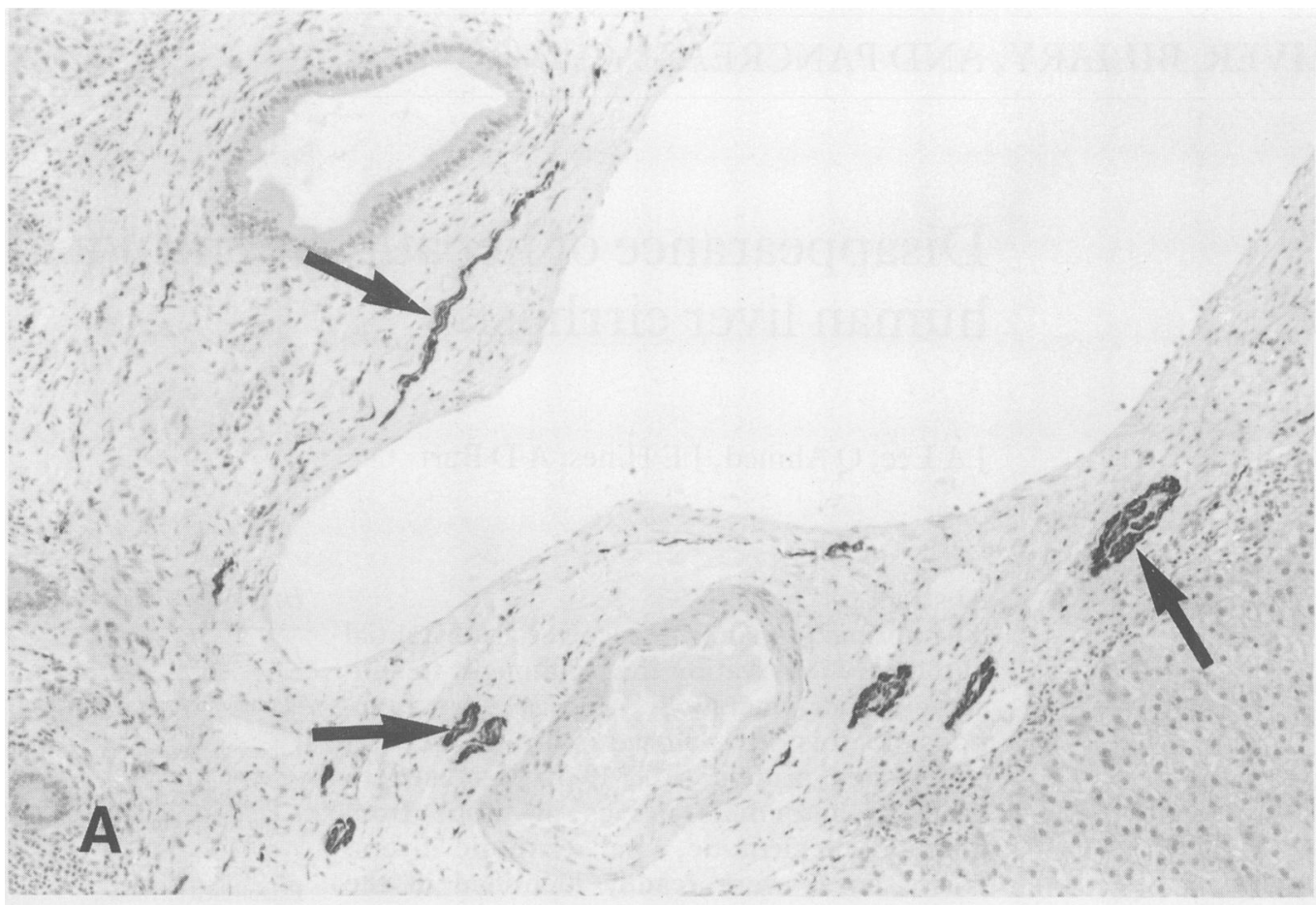

Fig $1 A$

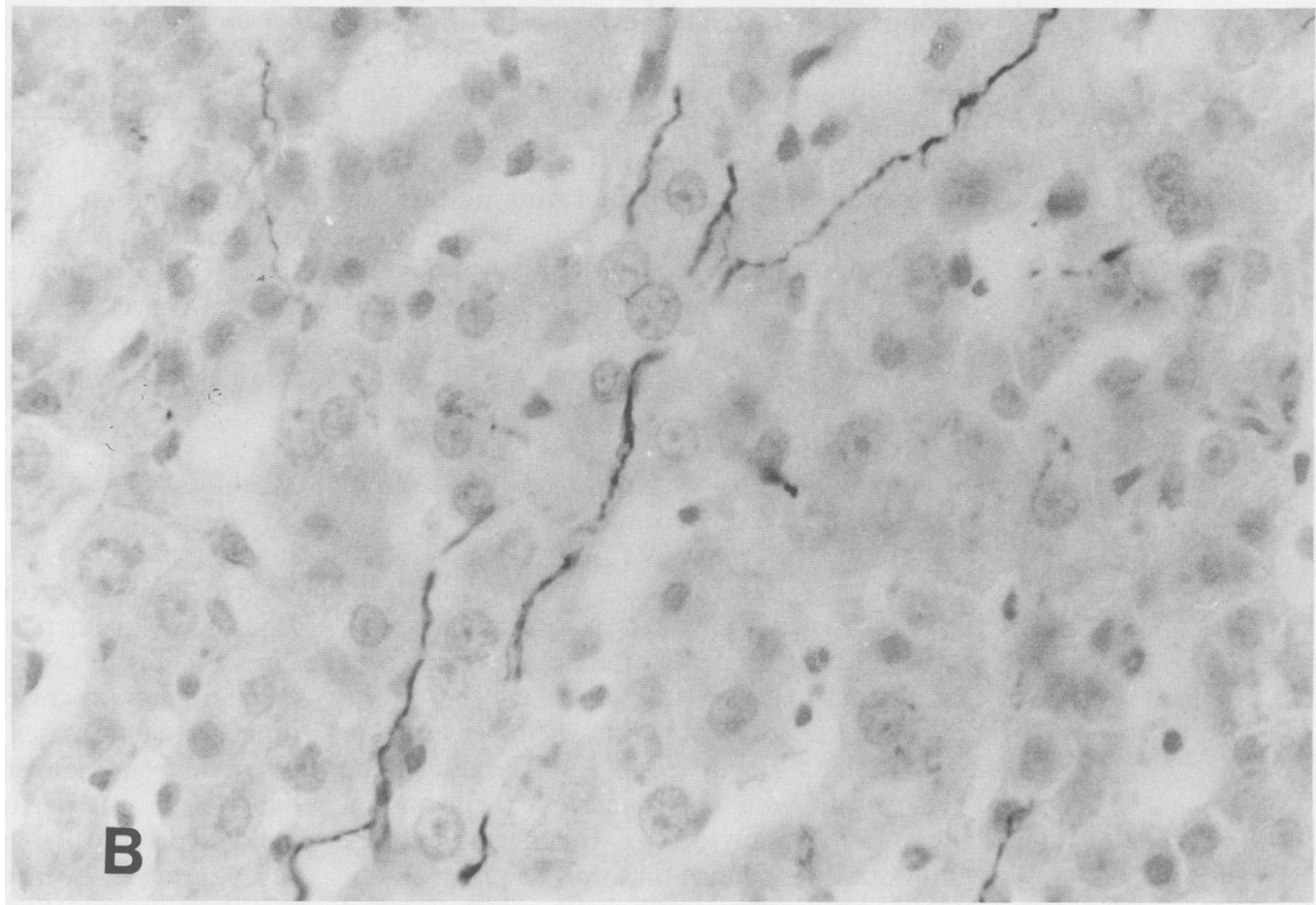

Fig $1 B$

then incubated with rabbit polyclonal anti-PGP $9 \cdot 5$ (Ultraclone, UK; dilution $1: 20$ in $20 \%$ NSS/ TBS), for 30 minutes at room temperature; (e) rinsed in TBS, then incubated with swine anti-rabbit immunoglobulin (Dako - dilution $1 / 20$ in NSS/TBS) for 30 minutes at room temperature, and finally incubated with peroxidase-antiperoxidase complex (Dakopatts, Denmark; dilution 1:50 in NSS/TBS) for 30 minutes at room temperature; ( $f$ ) rinsed in TBS, then incubated with 3,3' diaminobenzidine in the presence of hydrogen peroxide; (g) washed in tap water, counterstained with haematoxylin and mounted in DPX. As negative controls, sections were incubated with normal rabbit serum in
NSS/TBS in place of anti-PGP 9.5; no staining

\section{Results}

NERVE FIBRES IN NORMAL LIVER

Nerve fibres and bundles were prominent within the portal tracts (Fig 1A). They were mainly identified closely applied to hepatic artery and portal vein branches and only occasionally around bile duct radicles. In confirmation of previous studies ${ }^{4}$ fibres were also seen within the liver parenchyma (Fig 1B). These fibres were seen to run along the sinusoidal walls within the was observed in these controls. 
Figure 2: Cirrhotic liver. (A) PGP 9.5

immunoreactive fibres are seen within the fibrous septo and residual portal tracts, but are absent from the regenerative nodules $(N)$ (original magnification $\times 20)$. (B) Higher power field shows absence of intrasinusoidal nerve fibres. Fibres are seen within the fibrous septum (arrow) (original magnification $\times 100)$.

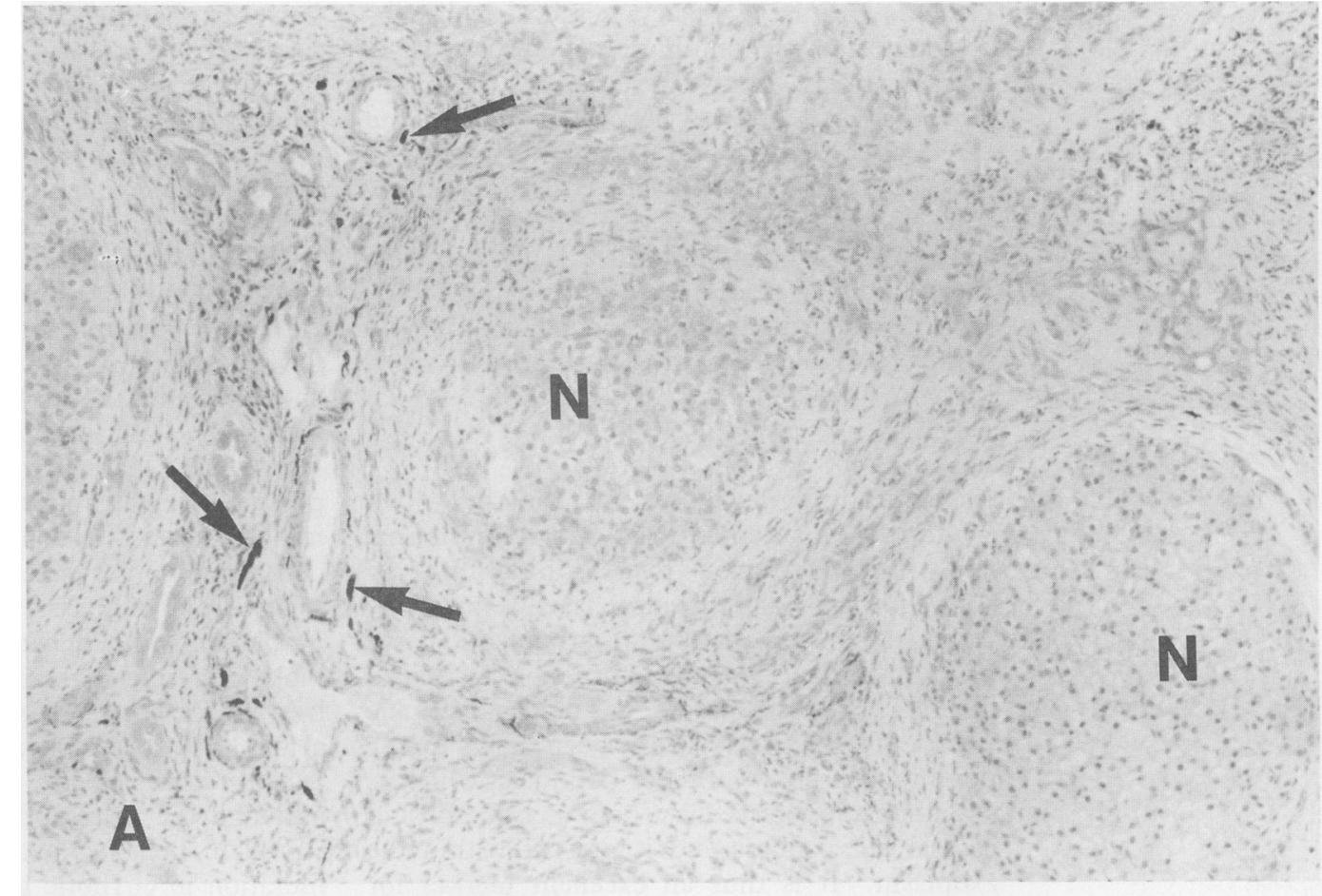

Fig $2 A$

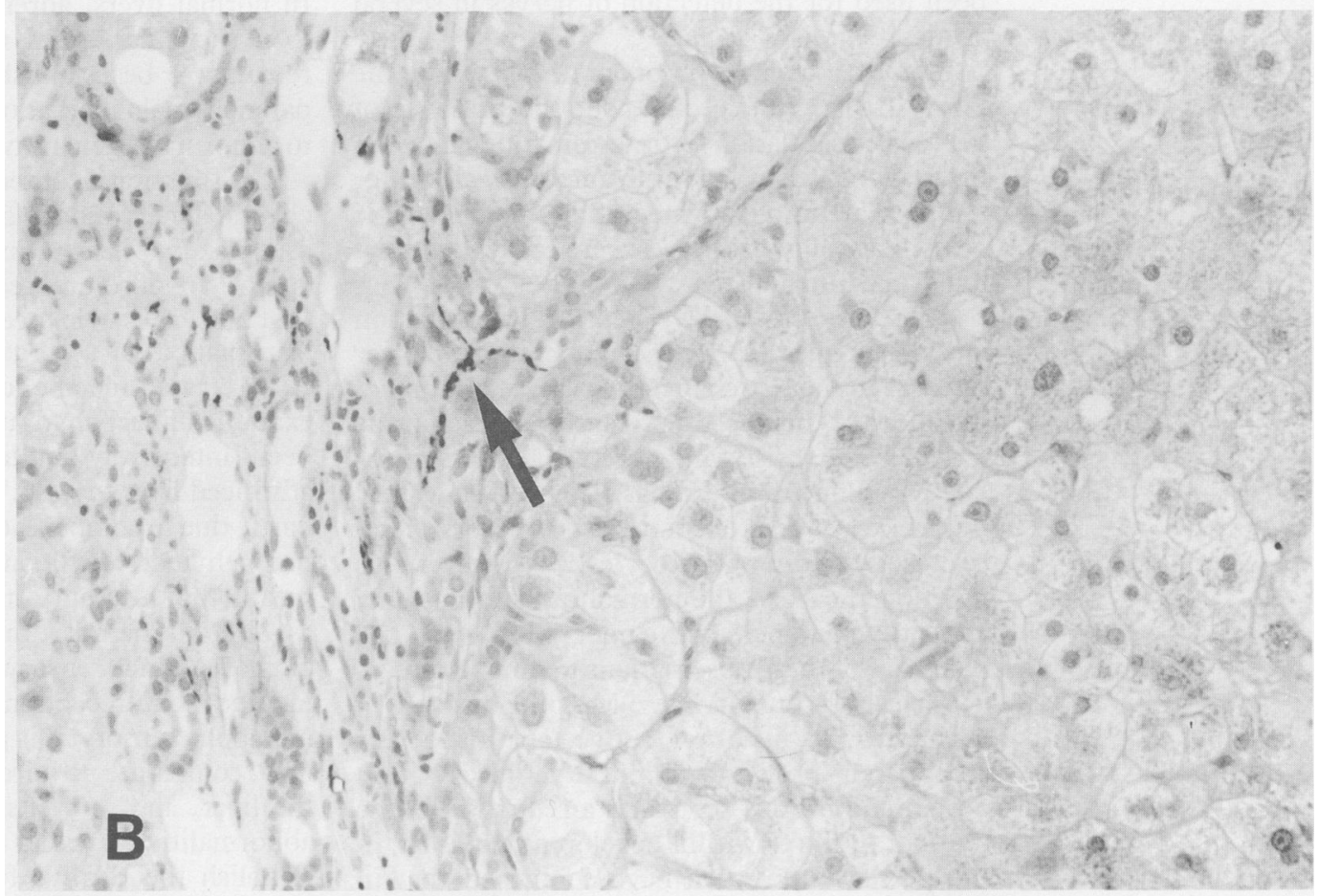

Fig $2 B$

space of Disse and were also observed between individual hepatocytes. The parenchymal nerves were generally most abundant within zone 1 (periportal) of the acinus, but were also frequently seen in zones II and III and within the walls of terminal hepatic vein branches.

NERVE FIBRES IN DISEASED LIVER

In wedge biopsy specimens showing non-specific reactive changes or evidence of acute cholestatic liver injury, the density and distribution of parenchymal nerve fibres were indistinguishable from normal livers. In the cases of acute hepatitis studied, there was an apparent increase in paren- chymal fibres, but the histological changes included the presence of appreciable bridging hepatic necrosis. In precirrhotic alcoholic liver disease and in stage I or II (precirrhotic) primary biliary cirrhosis there was a reduction in the numbers of parenchymal nerve fibres seen. In two cases of alcholic hepatitis and three cases of early primary biliary cirrhosis, only occasional fibres could be observed in periportal zones. In the remaining six cases of precirrhotic chronic liver disease, parenchymal fibres could be identified but were much less abundant than normal liver. The most dramatic change, however, was seen in established cirrhosis. Irrespective of the aetiology of the cirrhosis, whether caused by 
alcohol, primary biliary cirrhosis, haemachromatosis, or autoimmune chronic liver disease, nerve fibres were absent from the regenerating nodules of hepatic tissue in all but three cases (Fig 2, A and B). An internal control was present in cirrhotic livers in that nerve fibres were readily identified within the fibrous septa separating regenerative nodules (Fig 2, A and B). Indeed, in many cases, nerves within the fibrous septa were particularly prominent, in spite of a complete absence of parenchymal fibres.

\section{Discussion}

In this study we have used an antibody to PGP $9 \cdot 5$ to localise autonomic nerve fibres in normal and diseased human livers. Initial studies? showed that with our material this antibody detected a greater number of intrahepatic nerve fibres in normal liver than antibodies to neurofilament, $\mathrm{S} 100$ protein, or neuron specific enolase. PGP 9.5 has recently been shown to be a ubiquitin carboxy terminal peptidase which is abundantly expressed by both peripheral and central nerve fibres and is present within the axoplasm. ${ }^{8}$ It is thus an excellent marker for small, possibly unsheathed neurons, and has been used for the detection of nerves in several tissues. $^{9}$

In normal liver we observed nerves within the portal tracts, as expected, but the presence of nerves in association with terminal hepatic vein branches suggests that some fibres may also enter the liver along the vena cava and major hepatic veins. Parenchymal nerves were easily identified in normal human livers.

There has previously been little information on the changes in hepatic innervation in liver disease. In the rat, changes in the distribution of intrahepatic nerves after carbon tetrachloride induced necrosis ${ }^{10}$ and partial hepatectomy ${ }^{11}$ have been noted, and it has also been suggested that nerves have a role in the control of regenerative activity in hepatocytes. ${ }^{12}$ However, as mentioned earlier, the normal rat liver has only a scanty parenchymal innervation, ${ }^{5}$ so the relevance of these observations to human disease is unclear. In human liver, one previous study ${ }^{13}$ has shown that in chronic active hepatitis there is an apparent proliferation of nerve fibres within developing fibrous septa and a reduction in the number of fibres within developing nodules. In the present study, we have confirmed that in developing cirrhosis caused by alcohol or primary biliary cirrhosis the numbers of parenchymal nerve fibres are reduced. We have also shown that in established cirrhosis, irrespective of aetiology, parenchymal nerve fibres are absent from regenerating hepatic nodules.

These observations raise two interesting questions: what are the causes of nerve fibre loss in cirrhosis and what are the possible functional consequences? The mechanisms responsible for the loss of parenchymal nerve fibres in cirrhosis are as yet undetermined. Miyazawa et $a l^{13}$ suggested that nerve fibres might degenerate in evolving cirrhosis as part of the primary liver injury. Our finding of an essentially normal distribution of parenchymal nerve fibres in various forms of acute liver injury suggests, however, that there are likely to be other mechanisms. The increased parenchymal nerve fibre density in our case of acute hepatitis with bridging necrosis was probably caused by the effects of parenchymal collapse rather than true nerve proliferation. In chronic liver disease, it is possible that parenchymal nerves may be damaged by progressive fibrosis within the space of Disse. It seems unlikely, however, that this is entirely responsible for the lack of parenchymal nerves in cirrhosis, in view of the relative abundance of nerves in the fibrous septa. A cardinal feature of established cirrhosis is the presence of regenerative parenchymal nodules, and it is possible that this abnormal mode of growth is an important factor contributing to sinusoidal denervation. We are currently studying the relative roles of fibrosis and nodular regeneration in parenchymal denervation using sequential biopsy specimens from patients with progressive liver disease (primary biliary cirrhosis and alcoholic liver disease) and quantitation of nerve density using image analysis. It seems that although there are sufficient growth factors to stimulate hepatocyte and sinusoidal cell proliferation in cirrhotic liver, ingrowth of nerves into the regenerative nodules is not stimulated. In normal livers, adrenergic innervation seems to play an important role in regeneration. ${ }^{12}$ It remains to be established whether the lack of parenchymal innervation in cirrhosis contributes to abnormal regenerative growth patterns.

The functional consequences of hepatic parenchymal denervation in cirrhosis also remain to be established. Clearly, intact hepatic innervation is not an absolute requirement for adequate function, as is evidenced by the success of liver transplantation as organ replacement therapy. Also gross metabolic derangements - for example of carbohydrate metabolism which has been intensively studied - are rare even in advanced liver disease. ${ }^{14}$ This may, however, be partly due to compensation by other organs. The parenchymal denervation seen in cirrhosis means that afferent - that is, sensory - as well as efferent neural function will be absent in cirrhosis. Since sensory functions are thought to include baroreception, osmoreception, and metabolic sensors, it is possible that denervation may contribute to circulatory disturbances in the liver, and to systemic fluid and electrolyte abnormalities. Further work will be necessary to establish the relative functional importance of changes in the parenchymal innervation in developing and established cirrhosis.

1 Sawchenko PE, Friedman MI. Sensory functions of the liver a review. Am F Physiol 1979; 236: R5-20.

2 Lautt WW. Hepatic nerves: a review of their functions and effects. Can J Physiol Pharmacol 1980; 58: 105-23.

3 Friedman MI. Hepatic nerve function. In: Arias IM, Jakoby WB, Popper H, Schacter D, Shafritz DA, eds. The liver: WB, Popper H, Schacter D, Shafritz DA, eds. The liver:
biology and pathobiology. New York: Raven Press, 1988.

4 Bioulac-Sage P, Lafon ME, Saric J, Balabaud C. Nerves and perisinusoidal cells in human liver. $\mathcal{F}$ Hepatol $1990 ; 10$ : 105 12.

5 Burt AD, Tiniakos D, MacSween RNM, Griffiths MR, Wisse E, Polak JM. Localization of adrenergic and neuropeptide tyrosine-containing nerves in the mammalian liver. Hepatology 1989; 9: 839-49.

6 Forssmann WG, Ito S. Hepatocyte innervation in primates. f Cell Biol 1977; 74: 299-313.

7 Burt $\mathrm{AD}$, Ahmed Q, Lee JA. Innervation of human hepatic sinusoids: alterations in liver disease. Hepatol Rep Lit Rev 1990; 20: Section 6.

8 Wilkinson KD, Lee SK, Deshpande S, Duerksen-Hughes P, Boss JM, Pohl J. The neuron-specific protein PGP 9.5 is a 
ubiquitin carboxyl-terminal hydrolase. Science 1989; 246: 670-3.

9 Wilson POG, Barber PC, Hamid QA. The immunolocalisation of protein gene product 9.5 using rabbit polyclonal and mouse monoclonal antibodies. Br $\mathcal{F}$ Exp Pathol 1988; 69: mouse monocional antibodies. Br
91-104. GY, Donath T. Changes of the peripheral

10 Ungvary GY, Donath T. Changes of the peripheral autonomic nervous system in altered inter

11 Pietroletti R, Chamuleau RAFM, Speranza V, Lygidakis NJ. Immunocytochemical study of the hepatic innervation in the rat after partial hepatectomy. Histochem $\mathcal{F} 1987 ; 19: 327-32$.
12 Aschrif S, Gillespie JS, Pollock D. The effects of drugs or denervation on thymidine uptake into rat regenerating liver. Eur f Pharmacol 1974; 29: 324-7.

13 Miyazawa Y, Fukuda Y, Imoto M, Koyama Y, Nagura $H$. Immunohistochemical studies on the distribution of nerve fibres in chronic liver disease. Am $\mathcal{F}$ Gastroenterol 1988; 83:

14 Alberti KGMM, Johnston DG, Taylor R. Carbohydrate metabolism in liver disease. In: Wright $R$, MillwardSadler GH, Alberti KGMM, Karran S, eds. Liver and
biliary disease. 2nd ed. London: Balliere-Tindall, 1985: biliary disease. 2nd ed. London: Balliere-Tindall, 1985: 